\title{
Behaviour of a birch plywood under various experimental conditions
}

\author{
Lydie Caetano $^{1}$, Bertrand Galpin² ${ }^{2}$ Vincent Grolleau ${ }^{1, a}$, and Jean-Damien Capdeville ${ }^{3}$ \\ ${ }^{1}$ Univ. Bretagne-Sud, EA 4250, LIMATB, 56100 Lorient, France \\ ${ }^{2}$ Ecoles Militaires de Saint- Cyr Coëtquidan, Laboratoire de Mécanique et Matériaux, 56381 Guer, France \\ ${ }^{3}$ GTT, Gaztransport et Technigaz, 78470 Saint Rémy-Lès-Chevreuses, France
}

\begin{abstract}
Liquid Natural Gaz -LNG- carriers must provide cryogenic reliability and safety for at least 40 years. Most of the insulation systems belong to the so-called membrane type, in which the "insulation complex" is composed of a metal membrane as a barrier, a plywood panel, insulation foam and a plywood panel glued to the inner hull. During construction and exploitation at cryogenic temperature, an impact of the membrane may occur and could lead to a LNG leakage. In order to evaluate this risk, and the role of the plywood in the perforation process of the membrane, a series of original impact tests are carried out at room and cryogenic temperatures on metal-plywood samples thanks to a specially designed catapult. In order to obtain a reliable numerical simulation of the impact, a series of impact and out of plane compression tests at room and cryogenic temperatures are carried out. Thanks to a design experiment, we identified the influence of the moisture content, from $2 \%$ up to $30 \%$, and of the temperature, from $-173{ }^{\circ} \mathrm{C}$ up to $+20^{\circ} \mathrm{C}$, on the strain rate sensitivity of the plywood.
\end{abstract}

\section{Introduction}

Liquefied Natural Gas shipping transport is receiving more and more attention. LNG carriers must provide cryogenic reliability and safety for at least 40 years. Most of the insulation systems belong to the so-called membrane type, in which the "insulation complex" is composed of a metal membrane as a barrier (1.2 mm thick, 304 grade stainless steel), a plywood panel (12 mm thick, crossply plywood made from 9 birch veneers birch wood), insulation foam (Reinforced PolyUrethane Foam), and a plywood panel glued to the inner hull. During construction and exploitation, an impact of the membrane may occur and could lead to a LNG leakage. In order to evaluate this risk, and the role of the plywood in the perforation process of the membrane, a series of original impact tests are carried out on metal-plywood samples. A catapult and an instrumented projectile are used to perform impact tests. The results of the first tests lead us to conclude that the plywood plays a great role in the absorption of the impact energy. In a second phase, the out of plane behaviour of the plywood is studied under various experimental conditions of moisture content (MC), strain rate and temperature. As some other works revealed it, temperature [1], MC [2] and strain rate [3] have a great effect on the wood behaviour. But a huge interaction between temperature, strain rate and moisture content influences the stress plateau level $[4,5]$. Our work focuses on these interactions during the out of plane compression of the tested birch plywood.

\footnotetext{
${ }^{a}$ Corresponding author: vincent.grolleau@univ-ubs.fr
}

\section{Impact tests}

This section focus on the experimental set-up and the experimental evidence of the role the plywood plays on the perforation process of the membrane.

\subsection{Experimental set-up}

The instrumented projectile is propelled thanks to a specially designed pneumatic catapult, Fig. 1. The pneumatic catapult consists of a pneumatic piston propelling the projectile with an available kinetic energy of $2200 \mathrm{~J}(5 \mathrm{~kg}$ at $30 \mathrm{~m} / \mathrm{s})$. The projectile is guided thanks to guiding rods.

The $2.5 \mathrm{~kg}$ projectile is composed of two parts, (i) the base block, which is instrumented with an accelerometer, (ii) the sensing projection, instrumented using strain gages in order to measure the impact force applied to the sensing projection end, Fig. 1, [6,7]. We use a pair of tensile half-bridge-embedded, opposite-mounted gauges so as to filter possible flexural strains. The signal is amplified using a quick A2 Vishay bay and recorded with an acquisition adapter (1-MHz and 16-bit digitizing). The sensing projection is covered by a so-called cap-impacting end, which protects the gages. 5 geometries of the impacting end, i.e. flat, dihedral, trihedral, hemispherical and plate edge are available, Fig. 1. Here, the presented tests are limited to the $6 \mathrm{~mm}$ thick, $90^{\circ}$ dihedral shape.

The membrane and the plywood-foam sandwich are simply put-up on the catapult bed and a blank-holder applies a $2 \mathrm{MPa}$ pressure load on the periphery of the membrane, along a $50 \mathrm{~mm}$ wide strip. The dimension of the membrane is $400 \times 400 \mathrm{~mm}, 270 \mathrm{~mm}$ thick.

For cryogenic temperature tests, the plywood-foam sandwich is soaked onto liquid nitrogen prior to the test and liquid nitrogen is continuously sprinkled on

This is an Open Access article distributed under the terms of the Creative Commons Attribution License 4.0, which permits unrestricted use, distribution, and reproduction in any medium, provided the original work is properly cited. 


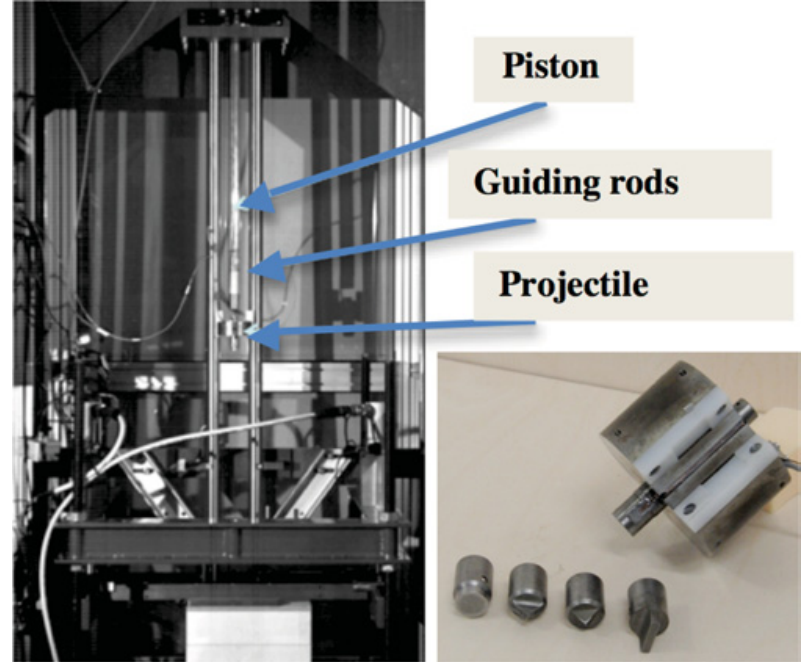

Figure 1. Pneumatic catapult (left side) and instrumented projectile along with some impacting ends (right side).
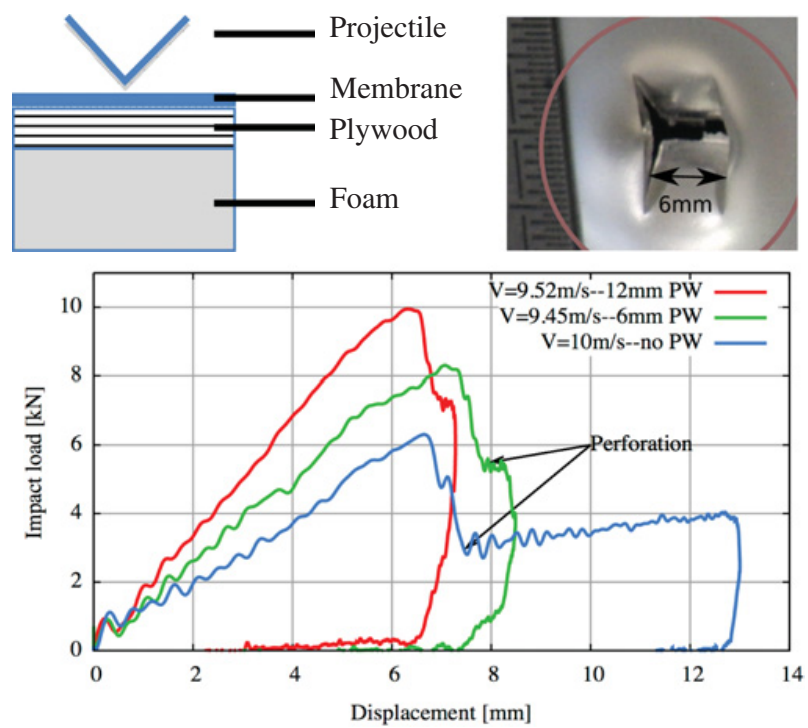

Figure 2. Impact load versus projectile displacement during dihedral impact of the insulation complex for different plywood thicknesses. Back side view of the perforated membrane when no plywood is used.

the membrane. The temperature is controlled using 3 thermocouples inserted onto the foam, the plywood or welded to the membrane.

\subsection{Impact test results}

In order to evaluate the role of the plywood in the perforation process, a series of impact test is performed with $12 \mathrm{~mm}$ thick, $6 \mathrm{~mm}$ thick or without plywood between the membrane and the foam. The presented results are obtained at ambient temperature, and the initial velocity of the $2.5 \mathrm{~kg}$, dihedral shape instrumented projectile is $9.7 \pm 0.5 \mathrm{~m} / \mathrm{s}$. Figure 2 shows the evolution of the impact load, namely the contact force between the membrane and the projectile, as a function of the solid displacement of the projectile. Thus, the area under the curve represents the amount of initial kinetic energy consumed during the impact. It is clear from Fig. 2 that the plywood influences drastically the perforation process of the membrane: when a $12 \mathrm{~mm}$ thick plywood is used, the perforation is not complete and the membrane deforms plastically until the projectile rebounds. Under the same conditions, a perforation occurs for $6 \mathrm{~mm}$ thick plywood and without plywood. The maximum impact load and the total lost in kinetic energy when the maximum impact load is reached are $40 \%$ higher in the case of $12 \mathrm{~mm}$ thick plywood than without plywood.

During the impacts, the plywood is mainly loaded under out of plane compression conditions. The lack of published results on this subject for the cryogenic conditions leads us to study more precisely the plywood behaviour under cryogenic and high strain rate conditions.

\section{Out of plane compression of the plywood}

The $12 \mathrm{~mm}$ thick cross-ply plywood is made from 9 birch veneers, which are peeled from logs. Thus the out of plane axis of the board refers to the radial direction of the wood $\log$. The compressive behaviour of the wood along this direction is known to be temperature, rate and moisture content sensitive, and well predicted from the theory of cellular solids [8].

\subsection{Experimental protocol}

A series of quasi-static out of plane compression tests have been performed with various diameters of specimens, from 12 up to $50 \mathrm{~mm}$, and various numbers of stacked samples, from 1 to 3 , in order to ensure that no size-effect would affect our results. In the following, a single $28 \mathrm{~mm}$ diameter circular sample is used.

The amount of moisture in wood,

$$
M C=\frac{m_{w e t}-m_{d r y}}{m_{d r y}}
$$

where $\mathrm{m}_{\text {wet }}$ is the mass of the specimen at a given moisture content and $\mathrm{m}_{d r y}$ is the mass of the ovendry specimen, is expressed as a percentage of wood mass when oven-dried [9].

In the following, the moistures contents are 2, 4, 8, $15 \%$ and fiber saturation point (FSP), about $28 \%$.

Quasi-static tests refer to the 4.e-4, 4.e-3, 4.e-2 and 4.e$1[/ \mathrm{s}]$ strain rate tests, while high strain rate tests refer to a $700 / \mathrm{s}$ strain rate.

In order to consider the variability due to the natural origin of the wood, each experimental condition is repeated at least five times. The curves presented Figs. 5 and 6 are the most representative ones of each series.

The temperatures of the tests refers to the initial temperature of the sample at the beginning of each test, and are in the $\left[-173^{\circ} ;+25^{\circ}\right]^{\circ} \mathrm{C}$ range. Every sample is radially drilled at mid span; the hole is $0.63 \mathrm{~mm}$ diameter and $9 \mathrm{~mm}$ long. These dimensions allow us to insert a Ttype thermocouple within the sample. The area of each sample is about $615 \mathrm{~mm}^{2}$ while the total area of this hole is less than $6 \mathrm{~mm}^{2}$, thus the behaviour of the sample is not affected by the hole. 


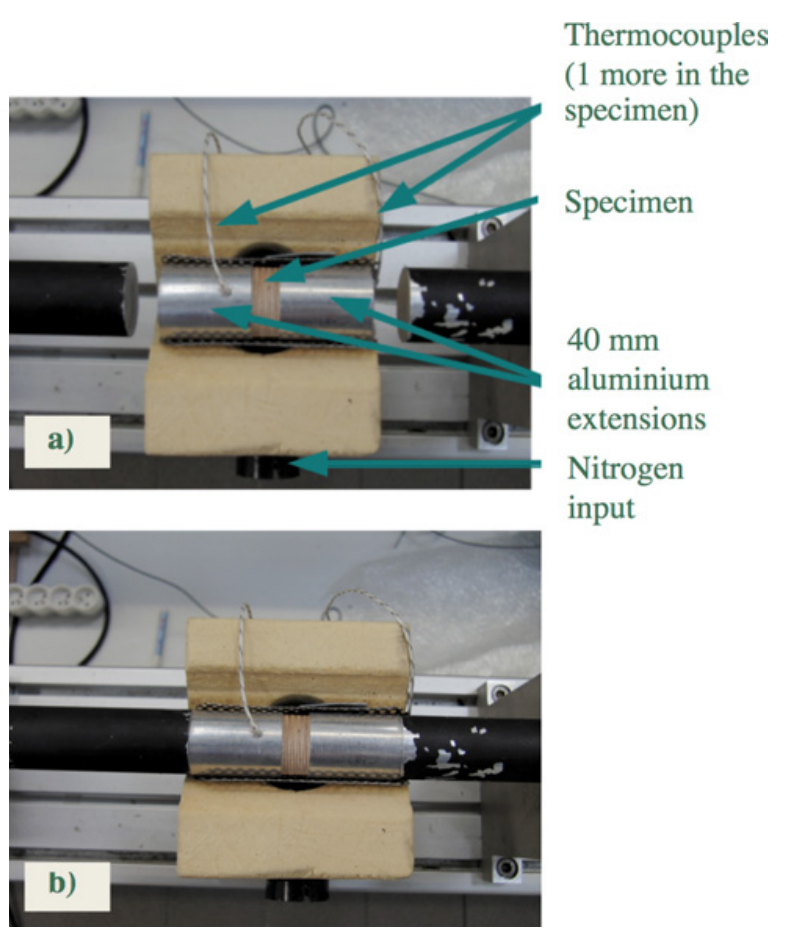

Figure 3. Extension rods - a) before contact $-b$ ) in contact.

\subsection{Quasi-static tests}

For quasi-static tests, a cryogenic chamber was conceived. Temperature is controlled thanks to 3 thermocouples located on the compression plateau, the chamber and the sample. A control loop regulates the temperature thanks to a remote valve and a liquid nitrogen sprinkler located on the top of the chamber. During the tests, the amplitude of variation of the initial temperature was found to be less than $\pm 10^{\circ} \mathrm{C}$.

\subsection{High strain rate SHPB tests}

For SHPB compression tests, $30 \mathrm{~mm}$ diameter aluminium bars and projectile are used. The $1 \mathrm{~m}$ long projectile impact generates a $400 \mu$ s incident signal duration and the initial speed, about $10 \mathrm{~m} / \mathrm{s}$, leads to a strain rate about 700/s.

For cryogenic tests, we decided to keep the bars at room temperature, and in order to avoid any warm-up of the sample when in contact with the bars, the sample is cool-down along with two $40 \mathrm{~mm}$ long $30 \mathrm{~mm}$ diameter aluminium extensions rods placed on the two faces of the sample, Figs. 3 and 4. Preliminary tests showed that the extensions do not affect the strain wave propagation even at cryogenic temperatures, and the only consequence is the modification of the strain-gage to bar end distances. Prior to the test, the sample and the two extension rods are placed in a climatic chamber. This specially designed chamber is made of polyurethane foam V-holder, and a foam cap. The axis of the chamber is coaxial to the bars axis and can move freely along this axis. A sprinkler cooldown the sample and the extension rods with a temperature regulated nitrogen gaz. During the tests, the temperatures of the injected nitrogen, the sample and the extension rods are recorded, the amplitude of variation of the initial temperature was found to be less than $\pm 5^{\circ} \mathrm{C}$.

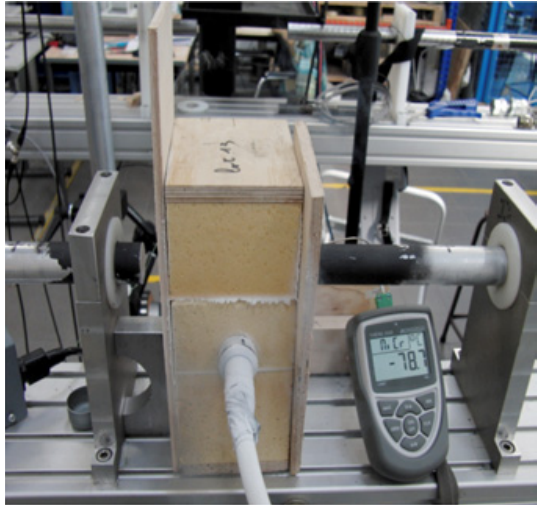

Figure 4. Cryogenic chamber completely close.

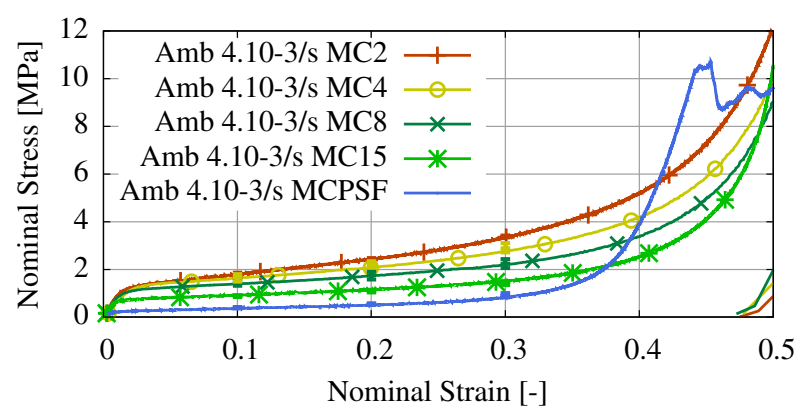

Figure 5. Engineering nominal stress versus nominal strain evolution for $12 \mathrm{~mm}$ thick birch plywood at $4 \mathrm{e}-3 \mathrm{~s}-1$ and room temperature for various moisture contents.

\section{Results}

Under quasi-static and ambient temperature conditions, the compressive behaviour of the $12 \mathrm{~mm}$ thick birch plywood follows the classic behaviour of cellular materials. The MC affects drastically the stress level: the higher the $\mathrm{MC}$, the lower the stress plateau value. The plywood behaviour can be described in three states: the elastic part, Fig. 5 until about 0.015 nominal strain at $8 \% \mathrm{MC}$, a stress plateau, Fig. 5 until about 0.45 strain at $8 \% \mathrm{MC}$, and the densification part, Fig. 5 over 0.45 strain at $8 \% \mathrm{MC}$.

The strain rate sensitivity of the plywood behaviour is huge. For example, for a $8 \% \mathrm{MC}$ and ambient temperature test, the engineering stress is $100 \%$ higher at 0.1 nominal strain and $120 \%$ higher at 0.2 strain at $700 / \mathrm{s}$ strain rate than for $4 . e-3 / s$. This difference exists under cryogenic temperature too and for each moisture contents, greater or less.

The cryogenic condition plays also a major role. For example, when the temperature falls from room to cryogenic conditions, for a $8 \% \mathrm{MC}$ and $4 . e-3 / \mathrm{s}$ strain rate test, the stress is $140 \%$ higher at 0.1 strain, $180 \%$ higher at 0.2 strain and $230 \%$ higher at 0.3 strain. It can be noticed that the evolution is not linear, as it is not linear for the effect of the strain rate too.

Under cryogenic and high strain rate conditions, it is clear from Fig. 6 that the interaction between temperature drop, MC and strain rate sensitivity reverse the MC effect on the stress plateau value: the higher the $\mathrm{MC}$, the higher the stress plateau value under cryogenic, $-173^{\circ} \mathrm{C}$, and high strain rate conditions about 700/s. 


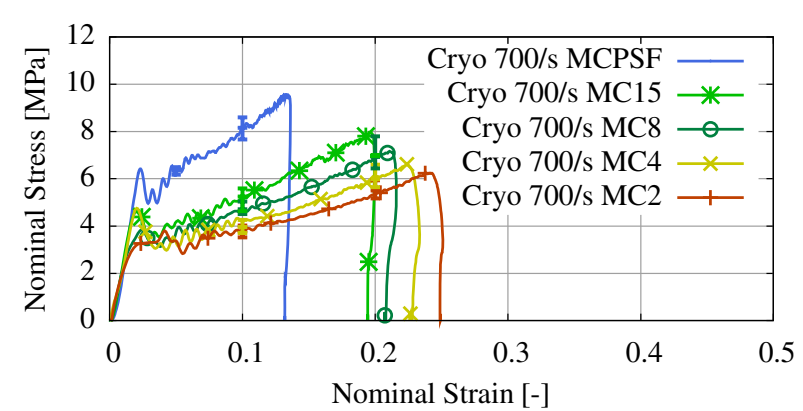

Figure 6. Engineering nominal stress versus nominal strain evolution for a $12 \mathrm{~mm}$ thick birch plywood at 700s-1 and cryogenic temperature for various moisture contents.

\section{Conclusion}

Impact tests have been performed on metal-plywood-foam complex. It has been proven that the plywood influences drastically the perforation process of the metal membrane. Some original compression tests have been done and different devices to impose moisture content, temperature and/or strain rate were developed. The main experimental result is the significant level of the strain rate, moisture content and temperature interactions on the stress plateau level during out of plane compression of this $12 \mathrm{~mm}$ thick birch plywood.

The results lead us to several conclusions on the effect of these three parameters on birch plywood behaviour and bring us experimental data to develop a numerical model.

\section{References}

[1] K.H. Boller, Strength of wood at low temperatures, Low temperature test methods and standards for containers, Committee on packing, packaging and preservation (December 1953)

[2] K. Siim, R. Kask, H. Lille and E. Täkker, 8th International DAAAM Baltic Conference, Study of physical and mechanical properties of birch plywood depending on moisture content, Ind. Eng. (2012)

[3] S.R. Reid and C. Peng, Dynamic uniaxial crushing of wood, IJIE, 19: 531-570 (1997)

[4] C.C. Gerhards, Effects of moisture content and temperature on the mechanical properties of wood: an analysis of immediate effects, W\&F, 14: 4-36 (1982)

[5] S. Widehammar, Stress-Strain Relationships for Spruce Wood: Influence of Strain Rate, Moisture Content and Loading Direction, SEM, 44: 44-48 (February 2004)

[6] B. Galpin, V. Grolleau, S. Umiastowski, G. Rio, and L. Mahéo. Design and application of an instrumented projectile for load measurements during impact, IJC, 13(2): 139-148 (2008)

[7] S. Tanimura. A new method for measuring impulsive force at contact parts, Expe. Mec., 24: 271-276 (1984)

[8] L.J. Gibson and M.F. Ashby. Cellular Solids Structure and Properties, Cambridge University Press, second edition (August 1999)

[9] Forest Service. Wood handbook, wood as an engineering material. Technical report, United States Department of Agriculture, Forest Service (2010) 